This item was submitted to Loughborough's Research Repository by the author.

Items in Figshare are protected by copyright, with all rights reserved, unless otherwise indicated.

\title{
After-exercise heart rate variability is attenuated in postmenopausal women and unaffected by estrogen therapy
}

\section{PLEASE CITE THE PUBLISHED VERSION}

http://dx.doi.org/10.1097/GME.0000000000000568

\section{PUBLISHER}

Lippincott, Williams \& Wilkins @ The North American Menopause Society

\section{VERSION}

AM (Accepted Manuscript)

\section{PUBLISHER STATEMENT}

This work is made available according to the conditions of the Creative Commons Attribution-NonCommercial 4.0 International (CC BY-NC 4.0) licence. Full details of this licence are available at: http://creativecommons.org/licenses/by-nc/4.0/

\section{LICENCE}

CC BY-NC 4.0

\section{REPOSITORY RECORD}

Harvey, Paula J., Emma ODonnell, Peter Picton, Beverley L. Morris, Catherine F. Notarius, and John S. Floras. 2019. "After-exercise Heart Rate Variability Is Attenuated in Postmenopausal Women and Unaffected by Estrogen Therapy”. figshare. https://hdl.handle.net/2134/20601. 


\title{
After-exercise heart rate variability is attenuated in postmenopausal women and unaffected by estrogen therapy
}

\author{
Paula J Harvey, BMBS, PhD, FRACP ${ }^{1,2}$, Emma O’Donnell, $\mathrm{PhD}^{2}$, \\ Peter Picton, MASc, $\mathrm{MA}^{2}$, Beverley L Morris, $\mathrm{RN}^{2}$, Catherine F Notarius, $\mathrm{PhD}^{2}$, \\ and John S Floras, MD, DPhil, FRCPC ${ }^{2,3}$.
}

Division of Cardiology at ${ }^{1}$ Women's College Hospital, ${ }^{2}$ University Health Network and ${ }^{3}$ Mount Sinai Hospital, Department of Medicine, University of Toronto, Toronto, Ontario, Canada.

\section{Corresponding Author}

Dr Paula J Harvey, BMBS, PhD, FRACP

Division of Cardiology, Women's College Hospital, Department of Medicine, University of Toronto, 76 Grenville Street

Toronto, Ontario, M5S 1B3

Canada

Tel: 416-323-7722

Fax: 416-323-6304

Paula.harvey@wchospital.ca

Running Title: Heart Rate Variability in Menopausal Women

Financial Disclosure/Conflicts of Interest: No conflicts of interest, financial or otherwise, are declared by the author(s).

Funding: This study was supported by Grants-in-Aid T4050 and T4938 from the Heart and Stroke Foundation of Ontario. Dr. Paula Harvey was the recipient of a National Health and Medical Research Council of Australia Neil Hamilton Fairley Postdoctoral Research Fellowship and a Heart and Stroke Research Corporation of Canada Research Fellowship. Dr. Floras holds the Canada Research Chair in Investigative Cardiovascular Biology and was a Career Investigator of the Heart and Stroke Foundation of Ontario. 


\begin{abstract}
Objective: Delayed heart rate (HR) recovery in the immediate post-exercise period has been linked to adverse cardiovascular prognosis. The after-effects of an acute bout of exercise on HR modulation in postmenopausal women and the influence of estrogen therapy are unknown. Methods: In 13 sedentary postmenopausal women (PMW: 54 \pm 2 years; mean \pm SEM), we assessed heart rate variability (HRV), an index of HR modulation, and the influence of estrogen therapy on HRV. HRV in the frequency domain was quantified during supine rest and again 60 minutes after treadmill exercise for 45 minutes at $60 \% \mathrm{VO}_{2}$ peak. PMW were studied before and after 4 weeks of oral estradiol. To obtain reference values for the after-effects of exercise on HRV in healthy young females, 14 premenopausal women (PreM) completed the identical exercise protocol. Results: Compared with PreM, PMW demonstrated lower high frequency (HF; vagal modulation) and total HRV $(\mathrm{P}<0.05)$ at rest. In PreM, all HRV values were similar before and after exercise. In contrast, in PMW after exercise, despite having identical HR to PreM, HF and total HRV were all lower (all $\mathrm{P} \leq 0.01$ ) compared with pre-exercise HRV values. Estrogen therapy had no effect on pre- or post-exercise values for HRV. Conclusions: When compared with PreM, PMW have identical HR but lower vagal HR modulation at rest and delayed HRV recovery after exercise. Estrogen does not restore baseline HRV or accelerate HRV recovery post-exercise, suggesting aging rather than estrogen deficiency per se may lower HRV in postmenopausal women.
\end{abstract}

Keywords: autonomic function, estrogen, exercise, heart rate variability, menopause 


\section{INTRODUCTION}

Fluctuations in heart rate (HR) reflect autonomic modulation of sino-atrial discharge ${ }^{1,2}$. In cardiovascular disease diminished heart rate variability (HRV) usually reflects increased sympathetic and decreased vagal tonic HR modulation. These autonomic perturbations have been associated with increased risk for adverse cardiovascular events and mortality even in asymptomatic individuals ${ }^{3,4}$.

Exercise has profound and complex effects on autonomic HR modulation ${ }^{5}$. Importantly, these effects depend on the temporal relationship to exercise plus the cumulative effects of exercise training on autonomic tone. For example, increased cardiac sympathetic tone and coincident parasympathetic withdrawal occur during acute exercise 5,6. An increased risk of death during and immediately following exercise has been attributed in part to both the magnitude and subsequent rate of restoration of normal autonomic balance ${ }^{7}$. In direct contrast to these acute effects of exercise, regular aerobic exercise training is cardioprotective, and associated independently with a reduction in cardiovascular events and total mortality ${ }^{8}$. These benefits include an increased predominance of parasympathetic heart rate modulation in the exercise trained vs. the untrained state ${ }^{9,10}$

Potentially adverse changes in neural modulation of HR have been shown to occur following both natural ${ }^{11,12}$ and surgical ${ }^{13}$ menopause, thus suggesting that endogenous estrogen deficiency may be a factor contributing to a decrease in HRV following menopausal transition. Regular exercise is recommended as a nonpharmacological intervention to reduce cardiovascular risk and improve cardiovascular risk factor profile in postmenopausal women ${ }^{14,15}$. One mechanism contributing to the cardioprotective benefit of exercise in this population may be an enhancement of parasympathetic HR modulation in the physically trained state ${ }^{16,17}$.

Several of the cardiovascular benefits associated with exercise training may be induced rapidly, possibly after a single bout of exercise ${ }^{18}$. In this regard, we have demonstrated previously a reduction in blood pressure and an improvement in endothelial-dependent flow-mediated dilation in postmenopausal women when measured one hour following treadmill exercise for 45 minutes at $60 \%$ of peak oxygen consumption ( $\mathrm{VO}_{2}$ peak) ${ }^{19,20}$. To our knowledge, the pattern and rate of HRV recovery in healthy sedentary PMW woman after a single bout of sub-maximal aerobic exercise has not been reported, but may be of clinical relevance, given the contrasting immediate versus delayed effects of exercise on autonomic modulation. Furthermore, whether HRV recovery after exercise in this population is influenced by treatment with estrogen is unknown. Thus, this study in sedentary postmenopausal women was designed to determine, using frequency domain HRV analysis: 1) the pattern and degree of recovery of tonic autonomic HR modulation measured 60 minutes after a single bout of submaximal dynamic exercise of a type and intensity commonly prescribed to enhance cardiovascular health; 2) whether autonomic modulation following exercise is influenced by concurrent administration of oral estrogen. To characterize the after-effects of exercise on autonomic modulation in a comparator group of healthy younger estrogen-replete females, normotensive premenopausal women were submitted to the identical protocol. 


\section{METHODS}

Participants: We have previously reported the relationship between estrogen therapy, blood pressure and vascular function both before and after an acute bout of dynamic exercise in these pre- and postmenopausal women ${ }^{19,20}$. However, thus far we have not evaluated the effects of an acute bout of exercise on their HRV. Postmenopausal women $(n=13)$ were deemed healthy as determined by medical history, physical examination, and screening hematologic and biochemical testing performed before study entry. All were sedentary (no more than 2hrs of physical activity per week for at least the previous 6 months), normotensive (seated clinic blood pressure [BP] of $\leq 140 / 90 \mathrm{~mm} \mathrm{Hg}$ ), nondiabetic, non-smokers, and on no medications. To be eligible, participants reported at least 12 months of amenorrhea with the uterus intact or a previous hysterectomy. Biochemical evidence (serum follicle-stimulating hormone concentration [FSH] of $\geq 20$ IU/L) confirmed menopausal status. No subject received hormone replacement therapy for at least 2 months before study entry.

To obtain reference HRV values to compare with postmenopausal women, 14 healthy premenopausal women were also investigated under identical experimental conditions. All premenopausal women described regular menstrual cycles, averaging 25 to 35 days. None were receiving any oral contraceptive formulations. Premenopausal women were studied during the follicular phase (estrogen relatively unopposed by progesterone) between days 5 to 13 of the menstrual cycle. Pregnancy was excluded by a negative $\beta$-human chorionic gonadotropin test.

The protocol was approved by our institutional Human Ethics Review Committee, and all participants provided signed written consent.

Protocol: All experiments were conducted in the Clinical Cardiovascular Physiology Laboratory of the Toronto General Hospital in the morning at a stable temperature between 22 and $24^{\circ} \mathrm{C}$. All participants were fasted overnight and avoided exercise, alcohol, and caffeine over the 24 hours before the study. Participants lay in the supine position. An antecubital vein was cannulated for blood sampling. Heart rate (HR) was determined from lead II of an electrocardiogram recorded continuously throughout the study. Arterial BP was measured noninvasively at 1- minute intervals from the left arm by an automatic cuff recorder (Dinamap Pro 100, Critikon LLC, Tampa, Fla). A respiratory belt encircled the upper abdomen.

After 10 minutes of quiet supine rest in a semi-dark room, baseline BP and HR were acquired during 7 minutes of spontaneous breathing. All participants were then exercised on a treadmill for a total of 45 minutes. To provide similar exercise loads, the speed and grade of treadmill exercise were adjusted to maintain a target HR recorded during exercise at $60 \%$ of each individual's peak oxygen consumption ( $\mathrm{VO}_{2}$ peak), as measured during a graded exercise test performed on a separate day. After exercise, participants resumed the resting supine position. Post-exercise measurements commenced at 60 minutes after exercise. In postmenopausal women, this protocol was replicated 4 weeks after treatment with open-labelled oral estradiol, $2 \mathrm{mg} /$ day. Participants were instructed to take this estrogen formulation at approximately the same time each morning, with the exception of the study day. After completion of the study protocol, postmenopausal women with a uterus in situ were prescribed medroxyprogesterone 
acetate 10-mg tablets once daily for a period of 12 days to convert the endometrium from the follicular to the secretory state.

Heart Rate Variability (HRV): Our approach to frequency domain analysis of HRV has been previously described ${ }^{21}$. Briefly, during spontaneous breathing, the ECG signal was sampled at $1000 \mathrm{~Hz}$ and was stored using LabView (National Instruments, Austin, TX) for subsequent analysis. R-R intervals were analyzed using fast Fourier transformation (FFT) to produce a spectral density curve showing a plot of the frequency of the cyclic components of variation in the R-R interval duration against the square root of their amplitude. A 7-minute data set comprising 2,048 data points was collected and subsequently divided into seven segments, each containing 512 points, with one-half overlapping of each segment. The linear trend in the data was subtracted from the data set in each segment, and a Blackman-Harris window was applied to minimize spectral leakage. Power spectra were obtained over a frequency range of $0.0098-0.5 \mathrm{~Hz}$, permitting the report of power spectra across very low frequency (VLF) 0.0098-0.05 Hz, low frequency (LF: a composite of both sympathetic and parasympathetic modulation), 0.05- $0.15 \mathrm{~Hz}$, and high frequency (HF: an index of parasympathetic modulation), 0.15$0.5 \mathrm{~Hz}$. Frequency domains were determined in absolute units $\left(\mathrm{ms}^{2}\right)$ and with $\log _{10}$ transformation. For all data sets, only stationary time series with $\leq 5 \%$ arrhythmia or artefact were used for analyses.

Peak Aerobic Consumption: On a separate day, peak oxygen consumption (VO2 peak) was measured directly by open-circuit spirometry during a standard graded cardiopulmonary exercise stress test.

Blood Sampling: Blood was collected from participants at baseline before exercise in premenopausal women and in postmenopausal women on both study days. Serum concentrations of FSH and $17 \beta$-estradiol were analyzed using commercially available radioimmunoassay kits (Boehringer, Mannheim, Germany).

Statistical Analysis: All variables are expressed as mean \pm standard error of the mean (SEM). All baseline HRV absolute data variables demonstrated a positively skewed distribution. Consequently, VLF, LF and HF were log-transformed (log10) to minimize the variance and normalize the distribution. For postmenopausal women, the average values at each phase of the study were compared by 2-factor repeated-measures analysis of variance (SPSS version 22.0; SPSS Inc., Chicago, IL) with estrogen and exercise as within-subject factors. The Student-Newman-Keuls method was used for pairwise, post hoc, multiple comparisons. In premenopausal women, paired $t$-tests were used to compare differences before and after exercise. The Student $t$-test was used for unpaired observations (premenopausal vs. postmenopausal women). All statistical tests were 2tailed with $P<0.05$ considered the threshold for significance.

\section{RESULTS}

Participants: Baseline physical characteristics of postmenopausal women were: mean age $54 \pm 2$ years, body mass index (BMI) $28.6 \pm 1.5 \mathrm{~kg} / \mathrm{m}^{2}$ and mean $\mathrm{VO}_{2}$ peak $1.33 \pm 0.01$ 
$\mathrm{L} / \mathrm{min}^{19,20}$. For premenopausal women, mean age was $28 \pm 1$ years, and mean BMI was $22 \pm 1 \mathrm{~kg} / \mathrm{m}^{2}$. Mean $\mathrm{VO}_{2}$ peak for this group was $1.89 \pm 0.02 \mathrm{~L} / \mathrm{min}$. These VO2peak values were $73 \%$ and $85 \%$ of predicted, respectively ${ }^{22}$. Each of these characteristics were significantly different $(P<0.05)$ between the groups.

Blood Sampling: As expected, baseline pre-estrogen serum measures of 17 beta-estradiol in postmenopausal women were lower $(P<0.001)$ compared with post-estrogen $(22 \pm 3.3$ vs. $998 \pm 220 \mathrm{pmol} / \mathrm{L}$, respectively) ${ }^{19,20}$. FSH (IU/L) was significantly reduced $(P<0.001)$ by estrogen therapy (78 \pm 3 vs. $37 \pm 6$, pre- versus post-estrogen, respectively). In the premenopausal group mean serum estradiol measured in the follicular phase was $187 \pm 28 \mathrm{pmol} / \mathrm{L}$.

Resting Heart Rate and Arterial Pressure: The BP and HR values of postmenopausal women appear in Table 1 . With estrogen resting systolic BP was unchanged, but diastolic blood pressure (DBP) and mean arterial pressure (MAP) were lower $(P<0.01)$. Despite these changes there were no significant differences between pre-estrogen and postestrogen baseline resting values for HR. Resting HR and BP of premenopausal and postmenopausal women were similar $(P>0.05)$.

Resting HRV: Baseline frequency domain measures of HRV in postmenopausal women before and after estrogen are shown in Table 1. At rest, postmenopausal women demonstrated similar LF compared with premenopausal women both pre- and postestrogen. In contrast, resting HF were lower $(P<0.05)$ in postmenopausal women both before and after estrogen when compared with premenopausal women.

Exercise: All participants completed the full 45-minute exercise protocol. In postmenopausal women, heart rates achieved during exercise were $116 \pm 4$ and $114 \pm 3$ beat/min pre-estrogen and post-estrogen, respectively ${ }^{19,20}$. These values corresponded to $101 \% \pm 1 \%$ and $100 \% \pm 1 \%$ of the target exercise HR. Premenopausal women achieved mean heart rate of $128 \pm 3$ beats/min which corresponded to $100 \pm 2 \%$ of the target exercise heart rate. Exercise increased systolic blood pressure (SBP) by $17 \pm 3$ mmHg before estrogen as compared with $9 \pm 4 \mathrm{mmHg}$ post-estrogen $(P=0.12)$. Exercise increased average SBP by $10 \pm 4 \mathrm{mmHg}$ in the premenopausal group $(P=0.15$ premenopausal vs. postmenopausal pre-estrogen).

After-Exercise Heart Rate and Arterial Pressure: One hour after exercise, in postmenopausal women, SBP and DBP were lower $(P<0.05)$ both before and after estrogen therapy (Table 1). One hour after exercise, in premenopausal women, SBP and DBP were unchanged $(P>0.05)$. HR post exercise was elevated $(P>0.05)$ and identical in the 2 groups ${ }^{19,20}$.

After-Exercise Heart Rate Variability: In postmenopausal women, two-way analysis of variance identified that exercise significantly lowered all indices of HRV, except LF/HF, which remained unaltered $(P>0.05$; Table 1$)$. In contrast, no significant main effect for estrogen therapy was detected. After-exercise, postmenopausal women pre-estrogen therapy demonstrated lower $(P<0.05) \mathrm{LF}$, HF and Total HRV compared with 
premenopausal women (Table 1). Estrogen therapy did not alter $(P>0.05)$ resting or postexercise frequency domain measures of HRV. In premenopausal women, one hour after exercise, despite a similar heart rate, all HRV measures were unchanged from preexercise values $(P>0.05)$.

\section{DISCUSSION}

This study had two aims. The first was to compare the after-effects of a single bout of dynamic exercise on cardiac autonomic HR modulation assessed by frequency domain analysis of HRV in healthy sedentary premenopausal and postmenopausal women. The second was to investigate the influence of exogenous estrogen on the post-exercise HRV responses in postmenopausal women. Consistent with previous literature ${ }^{11,17,23,24} \mathrm{HF}$ spectral power, an index of parasympathetic modulation of heart rate, was lower at rest in postmenopausal compared with premenopausal women. Our key new findings were: 1) in healthy sedentary premenopausal women, frequency-domain indexes of heart rate modulation recorded one hour post submaximal dynamic exercise were not significantly reduced from pre-exercise values. In contrast to these findings in premenopausal women, in postmenopausal women; 2) HF and LF remained suppressed below pre-exercise values at one hour post acute exercise; and 3) pre-treatment with chronic oral estrogen did not augment vagal modulation at rest, or enhance HRV recovery in the post-exercise period, suggesting delayed recovery of autonomic modulation. In view of recent concerns that these indices of HRV are simply a function of $\mathrm{HR}^{25}$, it is important to emphasize that these differences between premenopausal and postmenopausal women occurred despite identical heart rates after exercise.

The present finding of attenuated HF power, an index of parasympathetic modulation of HR, in postmenopausal women at baseline rest when compared with a comparator group of premenopausal women is concordant with that of other investigators who have shown reductions in HRV in women following both natural ${ }^{11,16,23,24}$ and surgical ${ }^{13}$ menopause. However, both aging and postmenopausal hormonal deficiency may contribute to changes in autonomic heart rate modulation in the postmenopausal period. We did not show any significant influence on resting heart rate or augmentation of resting HRV in our postmenopausal women following administration of oral estrogen, suggesting that the difference in HRV observed between pre- and postmenopausal women may be more consistent with aging than estrogen deficiency.

Previous studies of the effects of exogenous estrogen on resting cardiac autonomic heart rate modulation in postmenopausal women have yielded inconsistent results. In some studies, exogenous estrogen has been shown to attenuate, although not necessarily fully reverse, menopause-associated decreases in HRV through enhancement of cardiac vagal and attenuation of cardiac sympathetic tone ${ }^{11,13,23,24}$ whereas other studies have been unable to replicate this finding ${ }^{26,27}$. These inconsistencies may reflect differences in subject baseline characteristics, in the type, dose, mode of administration and timing of exogenous estrogen and in the experimental condition. For example, Neves et al ${ }^{24}$ found that estrogen partially alleviated the decline in HRV in their postmenopausal women but only when acquired in the seated but not in the supine position.

Importantly, these previous investigators determined HRV in postmenopausal women pre and post-estrogen only under resting conditions. Little is known of the 
influence of exogenous estrogen on HRV following different stimuli known to influence autonomic heart rate regulation. For the purpose of this analysis we were particularly interested in the after-effects of a single bout of dynamic exercise. Exercise has complex effects on sympathovagal heart rate modulation. Regular physical activity is associated with increased HRV and in particular a shift towards enhanced parasympathetic modulation. These changes are believed to contribute to improved cardiac electrical stability, and thus to the well documented cardioprotective benefits of exercise training ${ }^{9}$, ${ }^{10}$. However, in contrast to these well recognized cardioprotective effects of regular physical activity, risk of sudden cardiac death is increased during and immediately after exercise $^{7,28}$. It is generally agreed that during exercise, there is parasympathetic withdrawal and sympathetic excitation ${ }^{5,6}$. Delayed heart rate recovery in the immediate post exercise period is postulated to reflect delayed parasympathetic recovery and has been shown to be a predictor of sudden cardiac death. However, data pertaining to the kinetics of recovery of autonomic function after cessation of exercise beyond the very immediate post-exercise period are much less well explored and the timing and pattern of the recovery of autonomic regulation back to baseline is debated ${ }^{6,18,29}$.

Of the relatively small number of studies examining the delayed effects of exercise on autonomic modulation as measured by HRV, the majority have been undertaken in young men. It is unclear whether parasympathetic withdrawal or alternatively sympathetic withdrawal predominates in the delayed post-exercise period in these studies. Intensity and type of exercise (e.g. interval vs. constant vs. resistance), timing and position of subject during acquisition of recordings post exercise vary between these studies and likely contribute to the heterogeneous findings in otherwise similar young male cohorts ${ }^{6,18,30-35}$.

There is no present published literature concerning HRV responses one hour after exercise in either healthy untrained premenopausal or postmenopausal women. Figueroa et al compared $\mathrm{HRV}$ at 20 minutes post walk exercise at $65 \% \mathrm{VO}_{2}$ peak in lean $(\mathrm{n}=8)$ and obese $(n=8)$ middle-aged women (40-60 years) with type 2 diabetes, and 12 obese women without type 2 diabetes ${ }^{36}$. These investigators reported suppressed HF, more marked in the obese and diabetic than lean and diabetic participants post-exercise. More delayed responses post-exercise were not studied, nor were women defined by menopausal status. We found no delayed post-exercise effect on HRV in our premenopausal women, but our postmenopausal women had persistently depressed HF and Total HRV values at one hour after acute submaximal walking exercise. Although we excluded participants with glucose intolerance, hyperlipidemia and hypertension, it is possible that the higher BMI and lower cardiorespiratory fitness of our postmenopausal women compared to premenopausal women contributed to the observed attenuated return of post-exercise HF to pre-exercise values. In support of this concept, previous studies report that elevated BMI is inversely and cardiorespiratory fitness positively associated with $\mathrm{HRV}^{37,38}$.

Limitations: We did not record HR during controlled breathing, but it has been demonstrated previously in middle-aged volunteers studied supine that paced and spontaneous breathing provide similar information if recording periods are short and if spontaneous breaths fall within the high-frequency band ${ }^{39}$ as it was in our participants both before and after exercise. 
Conclusions: In summary, we have shown that in sedentary premenopausal women HRV power spectra density recovers to pre-exercise values when measured an hour after a single bout of treadmill exercise performed at a submaximal intensity that is comparable to prescriptions recommended for maintenance of cardiovascular health. In contrast, postmenopausal women exhibit significant reductions in HF, LF and total HRV spectral power after exercise. This sustained attenuation, particularly of tonic vagal heart rate modulation, is not an artefact of HR, since heart rates after exercise were similar in the pre- and postmenopausal women. Four weeks of estrogen replacement did not increase resting HRV and did not accelerate post-exercise HRV recovery in postmenopausal women. These observations suggest aging, and mechanisms consequent to the accretion of other age-related changes, such as a high BMI and low cardiorespiratory fitness, contribute to the lower resting HRV and delayed HRV recovery one hour post exercise in our postmenopausal women.

Low resting HRV is a recognized marker of adverse prognosis in cardiac patients and healthy populations (Tsuji, 1996; Dekker, 2000). During, and recovery from, exercise is suggested to be a vulnerable window for dysrhythmias and fatal events (Lahiri 2008). Autonomic evaluation of postmenopausal women during recovery from exercise may therefore also be of prognostic importance, particularly in light of current directives to actively encourage this population to engage in physical activity as part of a healthy lifestyle (Manson 2002; Manson 1999). Thus, delineation of the additional information that could be yielded from post-exercise recovery of HRV in response to both acute and chronic exercise training, and how estrogen influences this process, is of significance to postmenopausal women. Understanding the short and long-term effects of exercise on the cardiac autonomic nervous system will provide insight into whether regular physical activity and improved fitness can over time positively influence the post-exercise recovery of vagal heart rate modulation of postmenopausal women.

\section{ACKNOWLEDGMENTS}

We appreciate the time and effort expended by the volunteer participants. We also thank the staff of the Clinical Cardiovascular Physiology Laboratory at the Toronto General Hospital, Toronto. 


\section{REFERENCES}

1. Kleiger RE, Stein PK, Bigger JT, Jr. Heart rate variability: measurement and clinical utility. Ann Noninvasive Electrocardiol. 2005;10(1):88-101.

2. Heart rate variability: standards of measurement, physiological interpretation and clinical use. Task Force of the European Society of Cardiology and the North American Society of Pacing and Electrophysiology. Circulation. 1996;93(5):1043-65.

3. Tsuji H, Larson MG, Venditti FJ, Jr., et al. Impact of reduced heart rate variability on risk for cardiac events. The Framingham Heart Study. Circulation. 1996;94(11):28505.

4. Dekker JM, Crow RS, Folsom AR, et al. Low heart rate variability in a 2-minute rhythm strip predicts risk of coronary heart disease and mortality from several causes: the ARIC Study. Atherosclerosis Risk In Communities. Circulation. 2000;102(11):1239-44. 5. Perini R, Veicsteinas A. Heart rate variability and autonomic activity at rest and during exercise in various physiological conditions. Eur J Appl Physiol. 2003;90(34):317-25.

6. Mourot L, Bouhaddi M, Tordi N, Rouillon JD, Regnard J. Short- and long-term effects of a single bout of exercise on heart rate variability: comparison between constant and interval training exercises. Eur J Appl Physiol. 2004;92(4-5):508-17.

7. Curtis BM, O'Keefe JH, Jr. Autonomic tone as a cardiovascular risk factor: the dangers of chronic fight or flight. Mayo Clin Proc. 2002;77(1):45-54.

8. Kokkinos P, Myers J. Exercise and physical activity: clinical outcomes and applications. Circulation. 2010;122(16):1637-48.

9. Melanson EL, Freedson PS. The effect of endurance training on resting heart rate variability in sedentary adult males. Eur J Appl Physiol. 2001;85(5):442-9.

10. Seals DR, Chase PB. Influence of physical training on heart rate variability and baroreflex circulatory control. J Appl Physiol (1985). 1989;66(4):1886-95.

11. Brockbank CL, Chatterjee F, Bruce SA, Woledge RC. Heart rate and its variability change after the menopause. Exp Physiol. 2000;85(3):327-30.

12. Rosano GM, Patrizi R, Leonardo F, et al. Effect of estrogen replacement therapy on heart rate variability and heart rate in healthy postmenopausal women. Am J Cardiol. 1997;80(6):815-7.

13. Mercuro G, Podda A, Pitzalis L, et al. Evidence of a role of endogenous estrogen in the modulation of autonomic nervous system. Am J Cardiol. 2000;85(6):787-9.

14. Manson JE, Greenland P, LaCroix AZ, et al. Walking compared with vigorous exercise for the prevention of cardiovascular events in women. N Engl J Med. 2002;347(10):716-25.

15. Manson JE, Hu FB, Rich-Edwards JW, et al. A prospective study of walking as compared with vigorous exercise in the prevention of coronary heart disease in women. $\mathrm{N}$ Engl J Med. 1999;341(9):650-8.

16. Jurca R, Church TS, Morss GM, Jordan AN, Earnest CP. Eight weeks of moderate-intensity exercise training increases heart rate variability in sedentary postmenopausal women. Am Heart J. 2004;147(5):e21.

17. Davy KP, DeSouza CA, Jones PP, Seals DR. Elevated heart rate variability in physically active young and older adult women. Clin Sci (Lond). 1998;94(6):579-84. 
18. Pober DM, Braun B, Freedson PS. Effects of a single bout of exercise on resting heart rate variability. Med Sci Sports Exerc. 2004;36(7):1140-8.

19. Harvey PJ, Morris BL, Kubo T, et al. Hemodynamic after-effects of acute dynamic exercise in sedentary normotensive postmenopausal women. J Hypertension. 2005;23(2):285-92.

20. Harvey PJ, Picton PE, Su WS, Morris BL, C.F. N, Floras.J.S. Exercise as an alternative to oral estrogen for amelioration of endothelial dysfunction in postmenopausal women. Am Heart J. 2005;149:291-7.

21. Ando S, Rahman MA, Butler GC, Senn BL, Floras JS. Comparison of candoxatril and atrial natriuretic factor in healthy men. Effects on hemodynamics, sympathetic activity, heart rate variability, and endothelin. Hypertension. 1995;26(6 Pt 2):1160-6.

22. Jones NL, Makrides L, Hitchcock C, Chypchar T, McCartney N. Normal standards for an incremental progressive cycle ergometer test. Am Rev Respir Dis. 1985;131(5):700-8.

23. Liu CC, Kuo TB, Yang CC. Effects of estrogen on gender-related autonomic differences in humans. Am J Physiol Heart Circ Physiol. 2003;285(5):H2188-93.

24. Neves VF, Silva de Sa MF, Gallo L, Jr., et al. Autonomic modulation of heart rate of young and postmenopausal women undergoing estrogen therapy. Braz J Med Biol Res. 2007;40(4):491-9.

25. Monfredi O, Lyashkov AE, Johnsen AB, et al. Biophysical characterization of the underappreciated and important relationship between heart rate variability and heart rate. Hypertension. 2014;64(6):1334-43.

26. Farag NH, Nelesen RA, Parry BL, Loredo JS, Dimsdale JE, Mills PJ. Autonomic and cardiovascular function in postmenopausal women: the effects of estrogen versus combination therapy. Am J Obstet Gynecol. 2002;186(5):954-61.

27. Fernandes EO, Moraes RS, Ferlin EL, Wender MC, Ribeiro JP. Hormone replacement therapy does not affect the 24-hour heart rate variability in postmenopausal women: results of a randomized, placebo-controlled trial with two regimens. Pacing Clin Electrophysiol. 2005;28 Suppl 1:S172-7.

28. Albert CM, Mittleman MA, Chae CU, Lee IM, Hennekens CH, Manson JE. Triggering of sudden death from cardiac causes by vigorous exertion. N Engl J Med. 2000;343(19):1355-61.

29. Goldberger JJ, Le FK, Lahiri M, Kannankeril PJ, Ng J, Kadish AH. Assessment of parasympathetic reactivation after exercise. Am J Physiol Heart Circ Physiol. 2006;290(6):H2446-52.

30. Heffernan KS, Kelly EE, Collier SR, Fernhall B. Cardiac autonomic modulation during recovery from acute endurance versus resistance exercise. Eur J Cardiovasc Prev Rehabil. 2006;13(1):80-6.

31. Parekh A, Lee CM. Heart rate variability after isocaloric exercise bouts of different intensities. Med Sci Sports Exerc. 2005;37(4):599-605.

32. Niemela TH, Kiviniemi AM, Hautala AJ, Salmi JA, Linnamo V, Tulppo MP. Recovery pattern of baroreflex sensitivity after exercise. Med Sci Sports Exerc. 2008;40(5):864-70.

33. Casonatto J, Tinucci T, Dourado AC, Polito M. Cardiovascular and autonomic responses after exercise sessions with different intensities and durations. Clinics. 2011;66(3):453-8. 
34. Seiler S, Haugen O, Kuffel E. Autonomic recovery after exercise in trained athletes: intensity and duration effects. Med Sci Sports Exerc. 2007;39(8):1366-73.

35. Raczak G, Pinna GD, La Rovere MT, et al. Cardiovagal response to acute mild exercise in young healthy subjects. Circ J. 2005;69(8):976-80.

36. Figueroa A, Baynard T, Fernhall B, Carhart R, Kanaley JA. Endurance training improves post-exercise cardiac autonomic modulation in obese women with and without type 2 diabetes. Eur J Appl Physiol. 2007;100(4):437-44.

37. Buchheit M, Gindre C. Cardiac parasympathetic regulation: respective associations with cardiorespiratory fitness and training load. Am J Physiol Heart Circ Physiol. 2006;291(1):H451-8.

38. Molfino A, Fiorentini A, Tubani L, Martuscelli M, Rossi Fanelli F, Laviano A. Body mass index is related to autonomic nervous system activity as measured by heart rate variability. Eur J Clin Nutr. 2009;63(10):1263-5.

39. Pinna GD, Maestri R, La Rovere MT, Gobbi E, Fanfulla F. Effect of paced breathing on ventilatory and cardiovascular variability parameters during short-term investigations of autonomic function. Am J Physiol Heart Circ Physiol. 2006;290(1):H424-33. 
Table 1. Pre- and post-exercise HR, BP and HRV in premenopausal women and healthy postmenopausal women pre- and post-estrogen therapy.

\begin{tabular}{|c|c|c|c|c|c|c|}
\hline & \multicolumn{2}{|c|}{ PreM (n=14) } & \multirow{2}{*}{\multicolumn{2}{|c|}{$\begin{array}{l}\text { PMW (n=13) } \\
\text { Pre Estrogen }\end{array}$}} & \multirow{2}{*}{\multicolumn{2}{|c|}{$\begin{array}{l}\text { PMW (n=13) } \\
\text { Post Estrogen }\end{array}$}} \\
\hline & & & & & & \\
\hline & Pre Ex & Post Ex & Pre Ex & Post Ex & Pre Ex & Post Ex \\
\hline SBP (mmHg) & $101 \pm 2$ & $98 \pm 2$ & $108 \pm 3$ & $103 \pm 2^{\mathrm{a}}$ & $106 \pm 3$ & $99 \pm 2^{\mathrm{a}}$ \\
\hline DBP (mmHg) & $61 \pm 2$ & $61 \pm 2$ & $64 \pm 2$ & $58 \pm 2^{\mathrm{a}}$ & $60 \pm 2^{b}$ & $56 \pm 2^{\mathrm{a}}$ \\
\hline MAP (mmHg) & $74 \pm 2$ & $73 \pm 2$ & $79 \pm 2$ & $73 \pm 2^{a}$ & $75 \pm 2^{b}$ & $70 \pm 2^{\mathrm{a}}$ \\
\hline HR (beats/min) & $59 \pm 2$ & $74 \pm 3 a$ & $61 \pm 3$ & $75 \pm 3^{\mathrm{a}}$ & $59 \pm 2$ & $74 \pm 3^{\mathrm{a}}$ \\
\hline LF $(\log 10)$ & $2.88 \pm 0.16$ & $2.76 \pm 0.15$ & $2.72 \pm 0.10$ & $2.08 \pm 0.12^{\mathrm{a}, \mathrm{c}}$ & $2.58 \pm 0.13$ & $2.09 \pm 0.10^{\mathrm{a}, \mathrm{c}}$ \\
\hline HF $(\log 10)$ & $3.08 \pm 0.16$ & $2.82 \pm 0.19$ & $2.64 \pm 0.12^{\mathrm{d}}$ & $2.11 \pm 0.17^{\mathrm{a}, \mathrm{c}}$ & $2.54 \pm 0.12^{\mathrm{d}}$ & $2.09 \pm 0.12^{\mathrm{a}, \mathrm{c}}$ \\
\hline VLF $(\log 10)$ & $2.90 \pm 0.11$ & $2.86 \pm 0.13$ & $2.85 \pm 0.12$ & $2.35 \pm 0.08^{\mathrm{a}, \mathrm{c}}$ & $2.74 \pm 0.09$ & $2.30 \pm 0.11^{\mathrm{a}, \mathrm{c}}$ \\
\hline
\end{tabular}

All values are mean \pm SEM.

DBP, diastolic blood pressure; HF, high frequency power spectra; HR, heart rate; LF, low frequency power spectra; log10, log 10 transformed data; MAP, mean arterial pressure; mmHg, millimetres of mercury; PMW, postmenopausal women; Pre Ex, pre-exercise; PreM, premenopausal women; Post Ex, post-exercise; SBP, systolic blood pressure; VLF, very low frequency power spectra.

${ }^{\text {a }} \mathrm{P}<0.05$ vs pre-exercise, within groups

${ }^{\mathrm{b}} \mathrm{P}<0.05$ vs PMW pre-exercise, pre-estrogen

${ }^{\mathrm{c}} \mathrm{P}<0.05$ vs PreM post-exercise

${ }^{\mathrm{d}} \mathrm{P}<0.05$ vs PreM pre-exercise 